\title{
A collaborative approach to training ward nurses in acute care skills in resource limited settings: the nursing intensive care skills training (NICTS) project
}

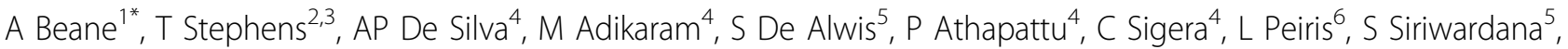 \\ KSA Jayasinghe ${ }^{7}$, A Dondorp ${ }^{8}$, R Haniffa $^{4,7,8}$
}

From ESICM LIVES 2015

Berlin, Germany. 3-7 October 2015

\section{Introduction}

Early recognition and prevention of deterioration of ward patients can improve patient outcomes and reduce critical care admissions [1]. In low and middle income countries (LMICs), with often minimal access to critical care therapies, the benefit may be even greater. However training to assist ward nurses develop acute care skills remains limited in such settings. As part of the NICST portfolio of acute care training, the Sri Lankan nursing faculty sought assistance to deliver a 2 day course for ward nurses [2].

\section{Objectives}

To design a clinically relevant short course for ward nurses in a LMIC to be delivered by local nursing tutors and facilitators.

To assess whether such a clinically focused programme would increase ward nurses' knowledge and skills in identifying and managing deteriorating patients.

\section{Methods}

A multi-modal 2 day acute care course for ward nurses was co-designed and delivered by specialist overseas trainers in partnership with national tutors. The courses were sponsored by the Ministry of Health, Sri Lanka. Based upon the NICST model of collaborative course design,

${ }^{1}$ Barts Health NHS Trust, Adult Critical Care Department, London, United Kingdom

Full list of author information is available at the end of the article local faculty were up skilled in delivery and content through a pre course Train the Trainer programme [3].

Candidates were invited to undertake on-line pre course e-learning. Core clinical guidelines were delivered using mini lectures. Facilitator-led skills stations and structured scenarios were used to develop clinical skills.

Short term knowledge acquisition was tested by a pre and post course Multi-Choice Questionnaire (MCQ). Newly acquired skills and their application was assessed through a post course Objective Clinical Skills Assessment (OSCA) station.

\section{Results}

122 ward nurses were trained over 6 courses in 2 locations. Post MCQ scores were significantly higher for each course compared to pre MCQ (Wilcoxon sign rank test $\mathrm{P}<0.0001$ ).

Over 71\% passed the OSCA (pass mark of 60). Feedback reveals high candidate satisfaction.

\section{Conclusions}

Our short course results demonstrate an increase in relevant knowledge and clinical skills of the participants. Our NICST model demonstrates the feasibility of a local nursing faculty in a LMIC co-designing and effectively delivering a setting adapted acute care training programme integrated into the local nurse training system.

\section{Authors' details}

${ }^{1}$ Barts Health NHS Trust, Adult Critical Care Department, London, United Kingdom. ${ }^{2}$ Barts Health NHS Trust, Critical Care Research Team, London, United Kingdom. ${ }^{3}$ Queen Mary University of London, William Harvey 
Table 1. Ward course pre and post course results

\begin{tabular}{|c|c|c|c|c|c|c|}
\hline Time & Venue & $\begin{array}{l}\text { Number of faculty members } \\
\text { trained }\end{array}$ & $\begin{array}{l}\text { Number of } \\
\text { trainees }\end{array}$ & $\begin{array}{l}\text { Pre Course MCQ mean } \\
\text { (SD) }\end{array}$ & $\begin{array}{l}\text { Post course MCQ mean } \\
\text { (SD) }\end{array}$ & $\begin{array}{l}P \\
\text { value }\end{array}$ \\
\hline 2014 June & Colombo & 21 & 31 & $66.3(14.8)$ & $80.3(11.2)$ & 0.0007 \\
\hline $\begin{array}{l}2014 \\
\text { September }\end{array}$ & Colombo & 20 & 14 & $53.2(9.3)$ & $67.4(9.9)$ & 0.0016 \\
\hline 2014 October & Moneragala & 16 & 27 & $51.0(14.4)$ & $73.3(11.8)$ & 0.0001 \\
\hline $\begin{array}{l}2014 \\
\text { November }\end{array}$ & Colombo & 13 & 13 & $52.0(7.0)$ & $65.3(8.6)$ & 0.0074 \\
\hline 2015 March & Moneragala & 11 & 20 & $46.8(10.6)$ & $65.8(7.9)$ & 0.0001 \\
\hline 2015 March & Colombo & 18 & 17 & 48.9 (14.6) & $67.6(12.7)$ & 0.0144 \\
\hline
\end{tabular}

Table 2. Selected course candidate feedback

\begin{tabular}{|c|c|c|c|c|c|c|}
\hline \multirow[t]{2}{*}{ Evaluation } & \multicolumn{6}{|c|}{ Strongly agree' and 'agree' combined } \\
\hline & $\begin{array}{l}\text { June } \\
(n=27)\end{array}$ & $\begin{array}{l}\text { Sept } \\
(\mathrm{n}=8)\end{array}$ & $\begin{array}{l}\text { Oct } \\
(n=23)\end{array}$ & $\begin{array}{l}\text { Nov } \\
(n=13)\end{array}$ & $\begin{array}{l}\text { March PBCN } \\
(n=11)\end{array}$ & $\begin{array}{l}\text { March M'ragala } \\
(\mathrm{n}=20)\end{array}$ \\
\hline I acquired new knowledge and skills & $27(100 \%)$ & $7(87.5 \%)$ & $22(95.7 \%)$ & $13(100 \%)$ & $11(100 \%)$ & $20(100 \%)$ \\
\hline $\begin{array}{l}\text { The sessions were supported with adequate } \\
\text { demonstration of intensive care skills }\end{array}$ & $25(96.1 \%)$ & $7(87.5 \%)$ & $22(100 \%)$ & $13(100 \%)$ & $11(100 \%)$ & $20(100 \%)$ \\
\hline The information in lectures was conveyed clearly & $23(85.2 \%)$ & $8(100 \%)$ & $21(95.5 \%)$ & $13(100 \%)$ & $11(100 \%)$ & $19(95 \%)$ \\
\hline I got adequate opportunity for hands-on experience & $24(92.3 \%)$ & $7(87.5 \%)$ & $20(90.9 \%)$ & $12(92.3 \%)$ & $11(100 \%)$ & $15(88.2 \%)$ \\
\hline I found the content relevant to my practice & $24(100 \%)$ & $7(87.5 \%)$ & $21(100 \%)$ & $13(100 \%)$ & $11(100 \%)$ & $20(100 \%)$ \\
\hline
\end{tabular}

Institute, London, United Kingdom. ${ }^{4}$ National Intensive Care Surveillance, Colombo, Sri Lanka. ${ }^{5}$ Ministry of Health, Office of Deputy Director General (Education, Training and Research), Colombo, Sri Lanka. ${ }^{6}$ Nursing Council of Sri Lanka, Colombo, Sri Lanka. 'University of Colombo, Department of Clinical Medicine, Faculty of Medicine, Colombo, Sri Lanka. ${ }^{8}$ Mahidol Oxford Tropical Medicine Research Unit (MORU), Bangkok, Thailand.

Published: 1 October 2015

\section{References}

1. Spearpoint KG, Gruber PC, Brett SJ: Impact of the Immediate Life Support course on the incidence and outcome of in-hospital cardiac arrest calls: an observational study over 6 years. Resuscitation 2009, 80(6):638-643.

2. Acute care training from Sri Lanka to the developing world. , NICS Training [Online] Available from: http://nics-training.com/?page_id $=507$ [Accessed 01:04:15].

3. De Silva AP, Stephens T, Welch J, Sigera C, De Alwis S, Athapattu P, et al: Nursing intensive care skills training: a nurse led, short, structured, and practical training program developed and tested in a resource-limited setting. J Crit Care 2015, 30(2):438.e7-11.

doi:10.1186/2197-425X-3-S1-A445

Cite this article as: Beane et al:: A collaborative approach to training ward nurses in acute care skills in resource limited settings: the nursing intensive care skills training (NICTS) project. Intensive Care Medicine

Experimental 2015 3(Suppl 1):A445.

\section{Submit your manuscript to a SpringerOpen ${ }^{\circ}$ journal and benefit from:}

- Convenient online submission

- Rigorous peer review

- Immediate publication on acceptance

- Open access: articles freely available online

- High visibility within the field

- Retaining the copyright to your article

Submit your next manuscript at $>$ springeropen.com 